\title{
The Penrose inequality for perturbations of the Schwarzschild initial data
}

\author{
J. Kopiński and J. Tafel \\ Faculty of Physics, University of Warsaw, \\ Pasteura 5, 02-093 Warsaw, Poland
}

\begin{abstract}
We show that in the conformally flat case the Penrose inequality is satisfied for the Schwarzschild initial data with a small addition of the axially symmetric traceless exterior curvature. In this class the inequality is saturated only for data related to special sections of the Schwarzschild spacetime.
\end{abstract}

\section{Introduction}

The mass $M$ and the surface area $\left|S_{h}\right|$ of the event horizon in the Kerr metric satisfy the inequality

$$
M \geq \sqrt{\frac{\left|S_{h}\right|}{16 \pi}}
$$

saturated by the Schwarzschild solution. Arguments based on the singularity theorems, no-hair theorems and thermodynamics of black holes led Penrose to the cosmic censorship conjecture and hypothesis that inequality (1) should be satisfied in physically realistic spacetimes on any initial surface $S$ with an internal horizon 11. Simultaneously Geroch 2, in his search for a proof of the positive energy theorem, proposed a definition of a quasilocal mass and showed its monotonicity under the inverse mean curvature flow (IMCF). This method was then pointed out by Jang and Wald [3] as a proper tool to prove (1) provided mathematical problems with singularities of IMCF would be resolved. Two decades later this approach was completed by Huisken and Ilmanen 4] under the assumption that the Ricci scalar of $S$ is positive and the internal horizon is a minimal surface. These conditions are natural if the initial data on $S$ are time symmetric. In more general setting the exterior curvature of $S$ should be admitted and the horizon would be a kind of a trapped surface. Some results in this direction were obtained by Malec, Mars and Simon [5] (see also [6]) who applied the Geroch method to the Hawking quasilocal mass. An extended discussion of results and ideas on the Penrose inequality can be found in a review by Mars [7.

In this paper we present an approach to the Penrose inequality based on the conformal method of solving constraints in general relativity [8, 9]. Initial data for the vacuum Einstein equations consists of a 3-dimensional Riemannian metric $g_{i j}^{\prime}$ and a symmetric tensor $K_{i j}^{\prime}$ constrained by the equations

$$
\begin{gathered}
\nabla_{i}^{\prime}\left(K^{\prime i}{ }_{j}-H^{\prime} \delta^{i}{ }_{j}\right)=0, \\
R^{\prime}+H^{\prime 2}-K^{\prime 2}=0,
\end{gathered}
$$


where $H^{\prime}=K^{\prime}{ }_{i}^{i}$ and $K^{\prime 2}=K^{\prime}{ }_{i j} K^{\prime i j}$. Let $S$ contains a 2-dimensional closed compact surface $S_{h}$, which is marginally outer trapped (MOTS),

$$
H^{\prime}-K_{n n}^{\prime}+2 h^{\prime}=0 \text {. }
$$

Here $K_{n n}^{\prime}=n^{\prime i} n^{\prime j} K^{\prime}{ }_{i j}, n^{\prime i}$ is the unit outer normal vector to $S_{h}$ and $h^{\prime}=$ $\frac{1}{2} \nabla^{\prime}{ }_{i} n^{\prime i}$ is the mean curvature of $S_{h}$. In order to find initial data admitting MOTS we start with preliminary data $g_{i j}, K_{i j}$ satisfying

$$
H=0
$$

and

$$
\nabla_{i} K^{i}{ }_{j}=0
$$

Then data

$$
g_{i j}^{\prime}=\psi^{4} g_{i j}, \quad K^{\prime i}{ }_{j}=\psi^{-6} K^{i}{ }_{j}, \quad \psi>0
$$

also satisfy (5)-(6) and the Hamiltonian constraint (3) is equivalent to the Lichnerowicz equation

$$
\triangle \psi=\frac{1}{8} R \psi-\frac{1}{8} K^{2} \psi^{-7},
$$

where $\triangle$ and $R$ are, respectively, the Laplace operator and the Ricci scalar related to $g_{i j}$. If $\psi$ satisfies in addition the boundary condition

$$
n^{i} \partial_{i} \psi+\frac{1}{2} h \psi-\frac{1}{4} K_{n n} \psi^{-3}=0 \text { on } S_{h}
$$

then the surface $S_{h}$ becomes MOTS with respect to the primed data. The existence theorems for equations (8)-(9) were proved by Dain [10] and Maxwell 11, 12] (see [13] for a review of results on solvability of the Lichnerowicz equation in different settings).

It is known that for some asymptotically flat data with MOTS inequality (1) is not true [14. A more plausible inequality follows if $\left|S_{h}\right|$ is replaced by a minimal area of surfaces enclosing $S_{h}$,

$$
M \geq \sqrt{\frac{A_{\min }\left(S_{h}\right)}{16 \pi}} .
$$

Moreover, as $S_{h}$ one can take an outermost MOTS

$$
M \geq \sqrt{\frac{A_{\min }\left(S_{\text {out }}\right)}{16 \pi}} .
$$

In the case of solutions to equations (8)-(9) we are not able to control if the resulting MOTS is outermost and if there are surfaces outside $S_{h}$ with a smaller area. Still, for a class of data we can prove (1) with just area of the MOTS. This inequality is stronger than (10) but may be weaker than (11). In fact we prove the stronger version of the Penrose inequality

$$
E^{2}-p^{2} \geq \frac{\left|S_{h}\right|}{16 \pi}+\frac{4 \pi}{\left|S_{h}\right|} J^{2},
$$

which makes sense in the case of axial symmetry. Here $E, p, J$ are, respectively, the total energy, momentum and angular momentum of data. 
In what follows we assume that data are axially symmetric and initial metric is conformally flat. In order to estimate components of 12 we study equations (8)- 9 perturbatively assuming expansions of $\psi$ into powers of a small parameter proportional to the preliminary exterior curvature. Our main conclusion is that in the conformally flat case the Penrose inequality 12 is preserved under a small addition of the axially symmetric traceless exterior curvature to the Schwarzschild initial data. Moreover, within this class inequality $\sqrt{12}$ is saturated only for 1-parameter spherically symmetric data which correspond to special slices of the Schwarzschild spacetime. The main value of our results is that, unlike in most approaches based on the monotonicity of the Geroch mass (except [5]), we do not assume that the internal horizon is a minimal surface.

The considered class of metrics cannot contain the Kerr metric since the latter does not admit a conformally flat slice. Still, our data admit nontrivial angular momentum $J$ which cannot be radiated away according to analysis in [15]. Thus, if metric tends to a stationary state without a naked singularity its end state should be just the Kerr metric with angular momentum $J$. Including the Kerr metric into our considerations would require a reformulation of our approach in order to avoid its dependence on exact solutions of the momentum constraint and on expansions into the spherical harmonics. An easier generalization seems to be admitting nonmaximal data $(H \neq 0)$, perhaps without axial symmetry, while still keeping the conformal flatness.

In section 2 we find convenient expressions for the total energy $E$ and the horizon area $\left|S_{h}\right|$ if the preliminary metric is flat and the boundary $S_{h}$ is the 2-dimensional sphere. In section 3 we apply these formulas to axially symmetric solutions of the momentum constraint. We expand the exterior curvature into the Legendre polynomials and find the minimal value of the quantity $P_{I}=$ $E^{2}-\left|S_{h}\right| / 16 \pi$ for a fixed value of $K_{n n}$ on $S_{h}$. It turns out that the Penrose inequality 12 is satisfied in the second order in $K_{i j}$ for all considered data except the generalized Bowen-York data [16, 17]. The latter need fourth order calculation which is done in section 4. Again, the Penrose inequality follows. This part is illustrated by a numerical simulation for a 2-parameter family of data.

\section{A change of total energy and the horizon area under the conformal transformation}

The construction of initial data via the conformal method with $H=0$ is relatively simple if the initial metric $g_{i j}$ is flat. Then one can solve explicitly the momentum constraint and express the exterior curvature in terms of arbitrary functions (see [18, 19] for the axially symmetric case and [20] for general one).

We assume that $g_{i j}=\delta_{i j}$ and the initial surface is given by $S=R^{3} \backslash B\left(0, \frac{m}{2}\right)$, where $B\left(0, \frac{m}{2}\right)$ is an open ball with radius $m / 2$ and the spherical boundary $S_{h}$. If $K_{i j}=0$ then solution of (8) and (9) reads

$$
\psi=\psi_{0}=1+\frac{m}{2 r}
$$

and transformation (7) leads to the Schwarzschild initial metric with mass $m$ and $S_{h}$ being a minimal surface. In this case the Penrose inequality is saturated. 
Our goal is to investigate how this inequality is affected by addition of a small amount of the exterior curvature to these data.

Let $K_{i j}$ be a traceless solution of the momentum constraint which is asymptotically flat in the sense that

$$
K_{i j}=O\left(r^{-2}\right), \quad \partial_{p} K_{i j}=O\left(r^{-3}\right) \text { if } r \longrightarrow \infty .
$$

Then $K_{i j}$ belongs to the weighted Sobolev space $W_{\rho-1}^{1,2}$, where $-1<\rho<0$. Now we can use results of Maxwell [11, 12, to show that equations (8)-(9) admit a solution $\psi$. From Proposition 3 in [11 and existence of transformation (9p) to the Schwarzschild data one obtains that the Yamabe constant is positive, $\lambda_{g}>0$ (this property follows also from the Sobolev inequality on $S$ proved in Theorem 6.2 in [21]). Now, existence of $(\psi-1) \in W_{\rho}^{2,2}$ for $\left.K_{n n}\right|_{S_{h}} \leq 0$ is assured by Theorem 4.2 in 12 and existence of $\psi$ for $2 h \geq\left. K_{n n}\right|_{S_{h}} \geq 0$ follows from Theorem 1 in 11 (note that we use definitions of $K_{i j}$ and $h$ with opposite signs). The upper limit in the latter case is not relevant for us since we will assume smallness of $K^{2}$. There are no existence theorems for $K_{n n}$ of indefinite sign on $S_{h}$.

In what follows we assume that $\psi$ exists and

$$
\psi \longrightarrow 1 \text { if } r \longrightarrow \infty \text {. }
$$

In order to extract an information about energy from (8)-(9) it is convenient to use tilded variables defined by

$$
\tilde{\psi}=\frac{\psi}{\psi_{0}}, \quad \tilde{g}_{i j}=\psi_{0}^{4} g_{i j}, \quad \tilde{K}_{i j}=\psi_{0}^{-2} K_{i j}
$$

Equations (8)- 9 are equivalent to

$$
\begin{gathered}
\tilde{\triangle} \tilde{\psi}=-\frac{1}{8} \tilde{K}^{2} \tilde{\psi}^{-7}, \\
\partial_{r} \tilde{\psi}=\frac{1}{16} \tilde{K}_{r r} \tilde{\psi}^{-3} \text { at } r=m / 2,
\end{gathered}
$$

where $\tilde{\triangle}$ is the Laplace operator corresponding to the Schwarzschild metric $\tilde{g}$. The ADM formula for the total energy of the ultimate data (7) yields

$$
E=m-\frac{1}{2 \pi} \lim _{r \rightarrow \infty} \oint_{S_{r}} r^{2} \tilde{\psi}_{, r} d \sigma,
$$

where $d \sigma$ is the surface measure on the unit sphere. Integrating (17) over $S$ with the volume element $\tilde{\eta}$ defined by $\tilde{g}$ one obtains

$$
E=m+\frac{1}{16 \pi} \int_{S} \tilde{K}^{2} \tilde{\psi}^{-7} \tilde{\eta}-\frac{m^{2}}{32 \pi} \oint_{S_{h}} \tilde{K}_{r r} \tilde{\psi}^{-3} d \sigma .
$$

Note that energy $E$ is finite thanks to conditions 14 and $(15)$.

Let $K_{i j}$ be proportional to a small parameter $\epsilon$. We assume that $\psi$ can be expanded into the sum

$$
\psi=\psi_{0}+\psi_{1}+\psi_{2}+\ldots
$$


where terms $\psi_{n}$ are of the order $\epsilon^{n}$. Hence

$$
\tilde{\psi}=1+\tilde{\psi}_{1}+\tilde{\psi}_{2}+\ldots
$$

and, up to $\epsilon^{2}$, equation 20 reads

$$
E=m+\frac{1}{16 \pi} \int_{S} \tilde{K}^{2} \tilde{\eta}-\frac{m^{2}}{32 \pi} \oint_{S_{h}} \tilde{K}_{r r}\left(1-3 \tilde{\psi}_{1}\right) d \sigma .
$$

In terms of untilded quantities formula (23) takes the form

$$
E=m+\frac{1}{16 \pi} \int_{S} K^{2} \psi_{0}^{-6} \eta-\frac{m^{2}}{128 \pi} \oint_{S_{h}} K_{r r}\left(1-\frac{3}{2} \psi_{1}\right) d \sigma,
$$

where $\eta$ is the flat volume element. An advantage of expression (24) over (19) is that to calculate its r. h. s. we don't need $\psi_{2}$. It is sufficient to find $\psi_{1}$ satisfying the flat Laplace equation

$$
\triangle \psi_{1}=0
$$

and the boundary conditions

$$
\begin{gathered}
\partial_{r} \psi_{1}+\frac{1}{m} \psi_{1}=\frac{1}{32} K_{r r} \text { at } r=m / 2, \\
\psi_{1}=0 \text { at } r=\infty .
\end{gathered}
$$

Up to $\epsilon^{2}$ the ultimate surface area of $S_{h}$ is

$$
\left|S_{h}\right|=\oint_{S_{h}} \psi^{4} r^{2} d \sigma=4 m^{2} \oint_{S_{h}}\left(1+4 \tilde{\psi}_{1}+4 \tilde{\psi}_{2}+6 \tilde{\psi}_{1}^{2}\right) d \sigma .
$$

In order to eliminate $\tilde{\psi}_{2}$ from 28 let us integrate an approximate version of equation (17) over spherical coordinates. Hence

$$
r^{-2} \psi_{0}^{-6}\left(r^{2} \psi_{0}^{2}\langle\tilde{\psi}\rangle_{, r}\right)_{, r}=-\frac{1}{8}\left\langle\tilde{K}^{2}\right\rangle,
$$

where

$$
\langle f\rangle=\oint_{S_{r}} f d \sigma
$$

for any function $f$. Twice integrating 29 over $r$ yields

$$
\langle\tilde{\psi}\rangle=\frac{1}{8} \int_{r}^{\infty} \frac{d r^{\prime}}{r^{\prime 2} \psi_{0}^{2}} \int_{\frac{m}{2}}^{r^{\prime}} \frac{\tilde{r}^{2}\left\langle K^{2}\right\rangle}{\psi_{0}^{6}} d \tilde{r}-\frac{c_{1}}{r \psi_{0}}+c_{2},
$$

where $c_{1}$ and $c_{2}$ are constants. Constant $c_{1}$ follows from integration of (18)

$$
c_{1}=\frac{m^{2}}{64}\left\langle K_{r r}\left(1-\frac{3}{2} \psi_{1}\right)\right\rangle_{h},
$$

where \langle\rangle$_{h}$ denotes the integral $30 p$ for $r=\frac{m}{2}$. Value

$$
c_{2}=4 \pi
$$


follows from the asymptotic condition $\psi \longrightarrow 1$.

Substituting (31) and (32) into (28) leads to

$$
\left|S_{h}\right|=16 \pi m^{2}+6 m^{2}\left\langle\psi_{1}^{2}\right\rangle_{h}-\frac{m^{3}}{4}\left\langle K_{r r}\left(1-\frac{3}{2} \psi_{1}\right)\right\rangle_{h}+2 m^{2} \int_{\frac{m}{2}}^{\infty} \frac{d r^{\prime}}{r^{\prime 2} \psi_{0}^{2}} \int_{\frac{m}{2}}^{r^{\prime}} \frac{r^{2}\left\langle K^{2}\right\rangle}{\psi_{0}^{6}} d r .
$$

The double integral in (33) simplifies if we change the order of integration (here $\theta$ is the Heaviside function)

$$
\int_{\frac{m}{2}}^{\infty} \frac{d r^{\prime}}{r^{\prime 2} \psi_{0}^{2}} \int_{\frac{m}{2}}^{\infty} \theta\left(r^{\prime}-r\right) \frac{r^{2}\left\langle K^{2}\right\rangle}{\psi_{0}^{6}} d r=\int_{\frac{m}{2}}^{\infty} d r \frac{r^{2}\left\langle K^{2}\right\rangle}{\psi_{0}^{6}} \int_{r}^{\infty} \frac{d r^{\prime}}{r^{\prime 2} \psi_{0}^{2}}
$$

Due to (34) formula (33) takes the form

$$
\left|S_{h}\right|=16 \pi m^{2}+6 m^{2}\left\langle\psi_{1}^{2}\right\rangle_{h}-\frac{m^{3}}{4}\left\langle K_{r r}\left(1-\frac{3}{2} \psi_{1}\right)\right\rangle_{h}+2 m^{2} \int_{\frac{m}{2}}^{\infty} \frac{r\left\langle K^{2}\right\rangle}{\psi_{0}^{7}} d r .
$$

With use of notation 30 expression 24 reads

$$
E=m-\frac{m^{2}}{128 \pi}\left\langle K_{r r}\left(1-\frac{3}{2} \psi_{1}\right)\right\rangle_{h}+\frac{1}{16 \pi} \int_{\frac{m}{2}}^{\infty} \frac{r^{2}\left\langle K^{2}\right\rangle}{\psi_{0}^{6}} d r .
$$

From $(35)$ and $(36)$ one obtains

$$
E^{2}-\frac{\left|S_{h}\right|}{16 \pi}=P_{I}
$$

where

$$
P_{I}=\left(\frac{m^{2}}{128 \pi}\right)^{2}\left\langle K_{r r}\right\rangle_{h}^{2}-\frac{3 m^{2}}{8 \pi}\left\langle\psi_{1}^{2}\right\rangle_{h}+\frac{m}{8 \pi} \int_{\frac{m}{2}}^{\infty} \frac{r^{2}\left(2-\psi_{0}\right)\left\langle K^{2}\right\rangle}{\psi_{0}^{7}} d r
$$

The Penrose inequality for the total ADM mass is satisfied up to the second order in the exterior curvature if

$$
P_{I} \geq \bar{p}^{2}
$$

where $\bar{p}$ is the total momentum given by

$$
p^{i}=\frac{1}{8 \pi} \lim _{r \rightarrow \infty} \oint r^{2} K_{r}^{i} d \sigma
$$

in the asymptotic Cartesian coordinates. If the sharp version of inequality 39 is satisfied then the Penrose inequality is true for a sufficiently small exterior curvature. If it is saturated we have to investigate higher order corrections.

\section{Axially symmetric perturbations}

If $H=0$ the momentum constraint in flat space equipped with the spherical coordinates $r, \theta, \varphi$ yields 21 ]

$$
K_{\varphi \theta}=\frac{\omega_{, r}}{\sin \theta}, \quad K_{\varphi r}=-\frac{\omega_{, \theta}}{r^{2} \sin \theta},
$$




$$
\begin{gathered}
\left(r^{3} K_{r r} \sin \theta\right)_{, r}+\left(r K_{r \theta} \sin \theta\right)_{, \theta}=0, \\
\left(K_{\theta \theta} \sin ^{2} \theta\right)_{, \theta}+\left(r^{2} K_{r \theta}\right)_{, r} \sin ^{2} \theta+r^{2} K_{r r} \sin \theta \cos \theta=0,
\end{gathered}
$$

where $\omega$ is an arbitrary function. The general solution of equations (42)-43) can be given explicitly in terms of one function [18, 19]. However, this description is not useful for our goals.

Let us consider regularity conditions which should be satisfied by $K_{i j}$. It follows from (41) that at $\theta=0, \pi$ derivatives of $\omega$ should vanish sufficiently fast,

$$
\omega_{, \theta} \sim \sin ^{3} \theta, \quad \omega_{, r} \sim \sin ^{4} \theta .
$$

Hence

$$
\omega=f \sin ^{4} \theta+J\left(\cos ^{3} \theta-3 \cos \theta\right)+c,
$$

where $f$ is a smooth function of $r$ and $z=\cos \theta, J$ is a constant which plays a role of the ADM angular momentum and $c$ is a constant which can be omitted. Moreover, components $K_{r r}$ and $K_{r \theta} / \sin \theta$ should be smooth and the following condition should be satisfied in a neighbourhood of the symmetry axis

$$
K_{\theta \theta} d \theta^{2}+K_{\varphi \varphi} d \phi^{2}=f_{1}\left(d \theta^{2}+\sin ^{2} \theta d \varphi^{2}\right)+f_{2} d(\cos \theta)^{2},
$$

where $f_{i}$ are smooth functions. Hence $K_{\theta \theta}$ should be smooth and

$$
K_{\theta}^{\theta}=K_{\varphi}^{\varphi}+f_{3} \sin ^{2} \theta .
$$

Since $H=0$ there is

$$
K_{\varphi}^{\varphi}=-K_{\theta}^{\theta}-K_{r}^{r}
$$

and equation (47) is equivalent to

$$
2 K^{\theta}{ }_{\theta}=-K_{r}^{r}+f_{4} \sin ^{2} \theta .
$$

If $K_{\theta \theta}$ is differentiable with respect to $z$ then 49 follows from (43). Thus, in addition to (45) it is sufficient to require that $K_{r r}, K_{\theta \theta}$ and $K_{r \theta} / \sin \theta$ are smooth functions of $r$ and $z$.

Equation (42) can be solved in terms of a potential $Q$,

$$
\begin{gathered}
K_{r \theta}=\frac{Q_{, r}}{r \sin \theta}, \\
K_{r r}=-\frac{Q_{, \theta}}{r^{3} \sin \theta} .
\end{gathered}
$$

Let us introduce a function $F$ such that

$$
K_{\theta \theta}+\frac{1}{2} r^{2} K_{r r}=F_{, z} .
$$

Then (43) yields an equation for $F$,

$$
\triangle_{s} F=\left(r Q_{, r}\right)_{, r}+\frac{1}{2 r}\left(1-z^{2}\right) Q_{, z z},
$$

where

$$
\triangle_{s} F=\left(\left(1-z^{2}\right) F_{, z}\right)_{, z}
$$

is the spherical Laplacian of $F$. 
The regularity conditions imply that $Q$ is everywhere smooth and

$$
Q_{, r} \sim\left(z^{2}-1\right) .
$$

Integrating (55) over $r$ yields

$$
Q=Q^{\prime}\left(z^{2}-1\right)+L(z),
$$

where functions $Q^{\prime}$ and $L$ are smooth. Function $L$ can be written in the form $L=L^{\prime}+a z+a^{\prime}$, where $a, a^{\prime}$ are constants and $L^{\prime}$ vanishes on the symmetry axis. Function $L^{\prime}$ can be incorporated in $Q^{\prime}$ and constant $a^{\prime}$ has no effect on $K_{i j}$. Without a loss of generality we can assume that

$$
Q=\left(z^{2}-1\right) q_{, z}+a z, \quad a=\text { const },
$$

where $q$ is a smooth function of $r$ and $z$. Substituting (57) into (53) yields

$$
\triangle_{s} F=\left(z^{2}-1\right) \partial_{z}\left(\left(r q_{, r}\right)_{, r}+\frac{1}{2 r} \triangle_{s} q\right) .
$$

Equation (58) is solvable with respect to $F$ if the integral of its r.h.s. over $z$ vanishes. Hence, $q$ should satisfy

$$
\int_{-1}^{1} z q d z=p r+\frac{\tilde{p}}{r}
$$

where $p, \tilde{p}$ are constants. Formula 40 shows that parameter $p$ is the value of the ADM momentum directed along the symmetry axis.

Another regularity condition for $q$ is related to the asymptotical flatness condition (14). It reads

$$
|q, r|<\text { const }<\infty .
$$

Note that under condition 60 function $F_{, z}$ will be also bounded.

The first two terms in expression (38) depend exclusively on the restriction of function $K_{r r}$ to $r=\frac{m}{2}$. Given it we would like to find the minimal value of the last term in $(38)$. This term has the form

$$
\frac{1}{2 \pi} \int_{\frac{m}{2}}^{\infty} \varrho r^{5}\left\langle K^{2}\right\rangle d r=P_{J}+\tilde{P}_{I},
$$

where

$$
\begin{gathered}
\varrho=\frac{m\left(1-\frac{m}{2 r}\right)}{4 r^{3}\left(1+\frac{m}{2 r}\right)^{7}} \\
P_{J}=2 \int_{\frac{m}{2}}^{\infty} d r \varrho r \int_{-1}^{1}\left(K_{\varphi \theta}^{2}+r^{2} K_{\varphi r}^{2}\right) \frac{d z}{1-z^{2}} \\
\tilde{P}_{I}=\int_{\frac{m}{2}}^{\infty} d r \varrho r \int_{-1}^{1}\left(2 r^{2} K_{r \theta}^{2}+2\left(K_{\theta \theta}+\frac{1}{2} r^{2} K_{r r}\right)^{2}+\frac{3}{2} r^{4} K_{r r}^{2}\right) d z .
\end{gathered}
$$

Since $\omega$ is not related to $K_{r r}$, the minimal value of $P_{J}$ is zero. In order to estimate minimum of $\tilde{P}_{I}$ let us decompose $q$ into the Legendre polynomials $P_{n}$

$$
q=\Sigma_{1}^{\infty} q_{n} P_{n},
$$


where

$$
q_{1}=\frac{3}{2}\left(p r+\frac{\tilde{p}}{r}\right)
$$

(see (59) and note that $q_{0}=0$ can be assumed) and other coefficients $q_{n}$ are unknown functions of $r$. Thanks to the standard property of the Legendre polynomials,

$$
\triangle_{s} P_{n}=-n(n+1) P_{n},
$$

from (51) one obtains

$$
K_{r r}=\frac{1}{r^{3}}\left(a+\Sigma_{1}^{\infty} n(n+1) q_{n} P_{n}\right)
$$

and

$$
\int_{-1}^{1} K_{r r}^{2} d z=\frac{2}{r^{6}}\left(a^{2}+\Sigma_{1}^{\infty} \frac{n^{2}(n+1)^{2}}{2 n+1}\left(q_{n}\right)^{2}\right) .
$$

From $(50)$ it follows that

$$
\int_{-1}^{1} K_{r \theta}^{2} d z=\frac{1}{r^{2}} \int q_{, r}\left(\left(z^{2}-1\right) q_{, z}\right)_{, z r} d z=\frac{2}{r^{2}} \Sigma_{1}^{\infty} \frac{n(n+1)}{2 n+1}\left(q_{n, r}\right)^{2} .
$$

Using again (67) and another identity,

$$
\left(z^{2}-1\right) P_{n, z}=\frac{n(n+1)}{2 n+1}\left(P_{n+1}-P_{n-1}\right),
$$

one can easily find a solution $F$ of (58). Hence

$$
K_{\theta \theta}+\frac{1}{2} r^{2} K_{r r}=\Sigma_{2}^{\infty}\left(\left(r q_{n, r}\right)_{, r}-\frac{1}{2} n(n+1) \frac{q_{n}}{r}\right) \tilde{P}_{n},
$$

where

$$
\tilde{P}_{n}=\partial_{z}\left(\triangle_{s}^{-1}\left(\left(z^{2}-1\right) \partial_{z} P_{n}\right)\right)=\frac{1}{n+2}\left(n P_{n}-\frac{2}{n-1} P_{n-1, z}\right), n \geq 2 .
$$

Polynomials $\tilde{P}_{n}$ are orthogonal

$$
\int_{-1}^{1} \tilde{P}_{k} \tilde{P}_{n} d z=c_{n} \delta_{k n}
$$

where

$$
c_{n}=\frac{2 n(n+1)}{(n-1)(n+2)(2 n+1)} .
$$

In order to show (74) let us assume that $k \leq n$. Then

$$
\int_{-1}^{1} P_{n-1, z} P_{k-1, z} d z=\left(P_{n-1} P_{k-1, z}\right)_{-1}^{1}
$$

and

$$
\int_{-1}^{1} P_{k-1, z} P_{n} d z=0,
$$


since polynomial $P_{k-1, z z}$ is of lower order than $P_{n-1}$ and $P_{k-1, z}$ is of lower order than $P_{n}$. From the standard identities

$$
\begin{gathered}
P_{k, z}=P_{k-2, z}+(2 k-1) P_{k-1}, \\
\int_{-1}^{1} P_{k} P_{n} d z=\frac{2}{2 n+1} \delta_{k n}
\end{gathered}
$$

one obtains

$$
\int_{-1}^{1} P_{n-1, z} P_{k} d z=\left(P_{n-1} P_{k}\right)_{-1}^{1}-\int P_{n-1} P_{k, z} d z=\left(P_{n-1} P_{k}\right)_{-1}^{1}-2 \delta_{k n} .
$$

Formulas (76)-788 lead to (74). Note that boundary terms present in 76 and 80 cancel each other due to the properties

$$
P_{k-1, z}( \pm 1)=\frac{1}{2}( \pm 1)^{k} k(k-1), \quad P_{k}( \pm 1)=( \pm 1)^{k} .
$$

It follows from 72 and 74 that

$$
\int_{-1}^{1}\left(K_{\theta \theta}+\frac{1}{2} r^{2} K_{r r}\right)^{2} d z=\Sigma_{2}^{\infty} c_{n}\left(\left(r q_{n, r}\right)_{, r}-\frac{1}{2} n(n+1) \frac{q_{n}}{r}\right)^{2} .
$$

Substituting (69), (70) and (82), with $q_{1}$ given by $(66)$, to $(64)$ yields

$$
\tilde{P}_{I}=\Sigma_{0}^{\infty} I_{n}
$$

where

$$
\begin{gathered}
I_{0}+I_{1}=3 \int_{\frac{m}{2}}^{\infty} \frac{\varrho}{r}\left(a^{2}+2 p \tilde{p}+5 \frac{\tilde{p}^{2}}{r^{2}}+5 p^{2} r^{2}\right) d r=\frac{a^{2}}{32 m^{2}}+\frac{p \tilde{p}}{16 m^{2}}+\frac{\tilde{p}^{2}}{8 m^{4}}+\frac{129 p^{2}}{128}, \\
I_{n \geq 2}=2 c_{n} \int_{\ln \frac{m}{2}}^{\infty} \varrho\left[\left(\ddot{q}_{n}-N q_{n}\right)^{2}+(N-1)\left(2 \dot{q}_{n}^{2}+3 N q_{n}^{2}\right)\right] d u
\end{gathered}
$$

and symbol $N$ and new coordinate $u$ are defined by

$$
N=\frac{1}{2} n(n+1), \quad r=e^{u}, \quad \dot{q}_{n}=\partial_{u} q_{n} .
$$

Let $F_{n \geq 2}$ be a space of functions $q_{n}$ with fixed value $q_{n h}$ at $r=\frac{m}{2}$ and $y_{n} \in F_{n}$ be a solution of equation following from the variational principle for $I_{n}$,

$$
\left(\partial_{u}^{2}-N\right)\left[\varrho\left(\partial_{u}^{2}-N\right) y_{n}\right]-2(N-1) \partial_{u}\left(\varrho \partial_{u} y_{n}\right)+3 N(N-1) \varrho y_{n}=0 .
$$

We will show that $I_{n}\left(y_{n}\right)$ is the absolute minimum of $I_{n}$ on space $F_{n}$. To this end we write a general function from $F_{n}$ as $q_{n}=y_{n}+q_{n}^{\prime}$, where $q_{n}^{\prime}=0$ at $r=\frac{m}{2}$. Then

$$
\begin{aligned}
& I_{n}\left(q_{n}\right)=I_{n}\left(y_{n}\right)+I_{n}\left(q_{n}^{\prime}\right)+ \\
& \quad 4 c_{n} \int_{\ln \frac{m}{2}}^{\infty} \varrho\left[\left(\ddot{q}_{n}^{\prime}-N q_{n}^{\prime}\right)\left(\ddot{y}_{n}-N y_{n}\right)+(N-1)\left(2 \dot{q}_{n}^{\prime} \dot{y}_{n}+3 N q_{n}^{\prime} y_{n}\right)\right] d u .
\end{aligned}
$$


If we remove derivatives of $y_{n}$ via integration by parts we see that the integral over $u$ in (88) disappears thanks to (87) and vanishing boundary values of $\varrho$ and $q_{n}^{\prime}$. Since $I_{n}\left(q_{n}^{\prime}\right) \geq 0$ we obtain

$$
I_{n}\left(q_{n}\right) \geq I_{n}\left(y_{n}\right)
$$

and this inequality is saturated only if $q_{n}=y_{n}$. This result also shows that solution of (87), if it exists, is unique (otherwise two different solutions, say $y_{n}$ and $y_{n}^{\prime}$, would have to satisfy $I_{n}\left(y_{n}^{\prime}\right)>I_{n}\left(y_{n}\right)$ together with $\left.I_{n}\left(y_{n}\right)>I_{n}\left(y_{n}^{\prime}\right)\right)$. Thus, solution of (87) is defined exclusively by the boundary value $y_{n h}=q_{n h}$ at $r=\frac{m}{2}$. Value of $I_{n}\left(y_{n}\right)$ is also given by boundary terms,

$$
I_{n}\left(y_{n}\right)=\frac{c_{n}}{32 m^{2}} y_{n h}\left(\ddot{y}_{n}-N y_{n}\right)_{h},
$$

but, in order to find $\ddot{y}_{n}$ at $r=\frac{m}{2}$, we have first to solve (87). Fortunately, using the symbolic programme Mathematica and method of trial end error we were able to find exact form of $y_{n}$,

$$
y_{n}=\frac{N y_{n h}}{2 N+1}\left(\frac{n-1}{n+1}\left(\frac{m}{2 r}\right)^{n+1}+\frac{n+2}{n}\left(\frac{m}{2 r}\right)^{n}\right) .
$$

It follows from 90 and 91 that

$$
I_{n}\left(y_{n}\right)=\frac{n^{2}(n+1)^{2}}{32 m^{2}\left(n^{2}+n+1\right)(2 n+1)} y_{n h}^{2} .
$$

Axially symmetric and asymptotically vanishing solution of equation 25 has the form

$$
\psi_{1}=\Sigma_{0}^{\infty} a_{n}\left(\frac{m}{2 r}\right)^{n+1} P_{n} .
$$

Coefficients $a_{n}$ follow from condition 26 ,

$$
a_{0}=-\frac{a}{4 m^{2}}, \quad a_{n \geq 1}=-\frac{n(n+1)}{4 m^{2}(2 n+1)} q_{n h} .
$$

Hence

$$
\left\langle\psi_{1}^{2}\right\rangle_{h}=\frac{\pi}{4 m^{4}}\left(a^{2}+\Sigma_{1}^{\infty} \frac{n^{2}(n+1)^{2}}{(2 n+1)^{3}} q_{n h}^{2}\right) .
$$

Taking into account equations (61), (83), 840, 889), (92) and (95), we obtain the following inequality for the quantity $P_{I}$ defined by (38)

$$
P_{I}-p^{2} \geq P_{J}+\frac{1}{32 m^{2}} \Sigma_{2}^{\infty} \frac{n^{2}(n+1)^{2}(n-1)(n+2)}{\left(n^{2}+n+1\right)(2 n+1)^{3}} q_{n h}^{2} .
$$

Here $P_{J}$ is given by $(63)$, so the r.h.s. of (96) is always nonnegative. Inequality (96) is saturated if $q_{n}=y_{n}$. If the angular momentum $J$ is nontrivial then $d \omega \neq 0$ and $P_{J}>0$. In order to estimate a lower bound of $P_{J}$ in terms of $J$ let us write expression 45 in the form

$$
\omega=\tilde{\omega}+J\left(z^{3}-3 z\right)+c, \quad \tilde{\omega}=f\left(1-z^{2}\right)^{2} .
$$


Then

$$
\begin{aligned}
& \int_{-1}^{1} \frac{\omega_{, z}^{2}}{1-z^{2}} d z=\int_{-1}^{1} \frac{\tilde{\omega}_{, z}^{2}}{1-z^{2}} d z-6 J \int_{-1}^{1} \tilde{\omega}_{, z} d z+9 J^{2} \int_{-1}^{1}\left(1-z^{2}\right) d z= \\
= & \int_{-1}^{1} \frac{\tilde{\omega}_{, z}^{2}}{1-z^{2}} d z+12 J^{2} .
\end{aligned}
$$

Hence

$$
P_{J} \geq \frac{J^{2}}{4 m^{2}}
$$

and this inequality is saturated if $f=0$. It is equivalent, up to $K^{2}$, to

$$
P_{J} \geq \frac{4 \pi}{\left|S_{h}\right|} J^{2}
$$

taking into account the expansion

$$
\frac{4 \pi}{\left|S_{h}\right|} J^{2}=\frac{J^{2}}{4 m^{2}}-\frac{J^{2}}{8 \pi m^{2}}\left\langle\psi_{1}\right\rangle_{h}-\frac{J^{2}}{16 \pi^{2} m^{2}}\left(2 \pi\left\langle\psi_{2}+\frac{3}{4} \psi_{1}^{2}\right\rangle_{h}-\left\langle\psi_{1}\right\rangle_{h}^{2}\right)+\ldots
$$

Substituting (100) into (96) written in terms of multipole moments of $K_{r r}$ yields

$$
E^{2}-p^{2} \geq \frac{\left|S_{h}\right|}{16 \pi}+\frac{4 \pi}{\left|S_{h}\right|} J^{2}+2^{-11} m^{4} \Sigma_{2}^{\infty} \frac{(n-1)(n+2)}{\left(n^{2}+n+1\right)(2 n+1)^{3}}\left(K_{r r}\right)_{h}^{2} .
$$

Inequality (102) is saturated if $f=0$ and $q_{n}=y_{n}$. If $\omega \neq J\left(z^{3}-3 z\right)+c$ or $q_{n} \neq y_{n}$ or one of the moments of $\left(K_{r r}\right)_{h}$ with $n \geq 2$ does not vanish then the sharp form of the strong Penrose inequality 12 is necessarily satisfied in the order $K^{2}$.

\section{Generalized Bowen-York initial data}

Inequality 102 does not lead to 12 only if $\omega=J\left(z^{3}-3 z\right)+c$ and $q=q_{1} z$. In this case tensor $K_{i j}$ is given by

$$
\begin{array}{r}
K_{r r}=\frac{a}{r^{3}}+3\left(\frac{\tilde{p}}{r^{4}}+\frac{p}{r^{2}}\right) \cos \theta, \quad K_{\theta}^{\theta}=K_{\varphi}^{\varphi}=-\frac{1}{2} K_{r r}, \\
K_{r \theta}=\frac{3}{2}\left(\frac{\tilde{p}}{r^{3}}-\frac{p}{r}\right) \sin \theta, \quad K_{\varphi r}=\frac{3 J}{r^{2}}\left(z^{2}-1\right), \quad K_{\varphi \theta}=0,
\end{array}
$$

where $a, p, \tilde{p}, J$ are constants. This form of $K_{i j}$ is equivalent to the generalized Bowen-York extrinsic curvature 17. Parameters $p$ and $\tilde{p} / m^{2}$ are the total momenta viewed, respectively, from $r=\infty$ and $r=0$ (the latter point can be thought as another infinity obtained by an inversion). The constant $a$ does not have any obvious physical interpretation.

In order to check the Penrose inequality in the case (103) we have to consider terms in the energy (20) and surface area (28) of the third order in $K$. These take the following form

$$
E^{(3)}=-\frac{7}{16 \pi} \int_{\frac{m}{2}}^{\infty} \frac{r^{2}}{\psi_{0}^{7}}\left\langle K^{2} \psi_{1}\right\rangle d r+\frac{3 m^{2}}{256 \pi}\left\langle K_{r r}\left(\psi_{2}-\psi_{1}^{2}\right)\right\rangle_{h},
$$




$$
\left|S_{h}\right|^{(3)}=16 m^{2}\left\langle\frac{\psi_{1}^{3}}{8}+\frac{3}{4} \psi_{1} \psi_{2}+\tilde{\psi}_{3}\right\rangle_{h} .
$$

Following derivation of (31) one obtains

$$
\left\langle\tilde{\psi}_{3}\right\rangle_{h}=-\frac{3 m}{128}\left\langle K_{r r}\left(\psi_{1}^{2}-\psi_{2}\right)\right\rangle_{h}-\frac{7}{8} \int_{m / 2}^{\infty} \frac{d r^{\prime}}{r^{\prime 2} \psi_{0}^{2}} \int_{\frac{m}{2}}^{r^{\prime}} \frac{\tilde{r}^{2}}{\psi_{0}^{7}}\left\langle K^{2} \psi_{1}\right\rangle d \tilde{r} .
$$

The third order correction to $(38)$ reads

$$
\begin{aligned}
P_{I}^{(3)}= & -\frac{7 m}{8 \pi} \int_{m / 2}^{\infty} \frac{r^{2}\left(2-\psi_{0}\right)\left\langle K^{2} \psi_{1}\right\rangle}{\psi_{0}^{8}} \mathrm{~d} r-\frac{m^{2}\left\langle K_{r r}\right\rangle_{h}}{1024 \pi^{2}} \int_{m / 2}^{\infty} \frac{r^{2}\left\langle K^{2}\right\rangle}{\psi_{0}^{6}} \mathrm{~d} r- \\
& -\frac{m^{2}}{4 \pi}\left\langle\frac{1}{2} \psi_{1}^{3}+3 \psi_{1} \psi_{2}\right\rangle_{h}-\frac{3 m^{4}}{16384 \pi^{2}}\left\langle K_{r r} \psi_{1}\right\rangle_{h}\left\langle K_{r r}\right\rangle_{h} .
\end{aligned}
$$

To compute it we need an explicit formula for a solution $\psi_{2}$ of the Poisson equation

with the boundary condition

$$
\Delta \psi_{2}=-\frac{1}{8 \psi_{0}^{7}} K^{2}
$$

$$
\partial_{r} \psi_{2}+\frac{1}{m} \psi_{2}=-\frac{3}{64} K_{r r} \psi_{1} \text { at } r=m / 2 .
$$

For data 103 we can expand both sides of the above equations into the Legendre polynomials. Only few first coefficients in $\psi_{2}$ survive and they can be found explicitly using the symbolic programme Mathematica. Surprisingly, expression $\left(P_{I}-\frac{4 \pi}{\left|S_{h}\right|} J^{2}\right)^{(3)}$ vanishes (we suppose that cancellation of terms in this expression has some deeper reasons, not known to us) and we have to pass to the fourth order calculus.

Then in place of (104)- 107) one obtains

$$
\begin{gathered}
E^{(4)}=\frac{7}{16 \pi} \int_{\frac{m}{2}}^{\infty} \frac{r^{2}}{\psi_{0}^{7}}\left\langle K^{2}\left(4 \frac{\psi_{1}^{2}}{\psi_{0}}-\psi_{2}\right)\right\rangle d r-\frac{m^{2}}{512 \pi}\left\langle K_{r r}\left(12 \psi_{1} \psi_{2}-6 \psi_{3}-5 \psi_{1}^{3}\right)\right\rangle_{h}, \\
\left|S_{h}\right|^{(4)}=4 m^{2}\left\langle\frac{\psi_{1}^{4}}{16}+\frac{3 \psi_{1}^{2} \psi_{2}}{2}+\frac{3 \psi_{2}^{2}}{2}+3 \psi_{1} \psi_{3}+4 \tilde{\psi}_{4}\right\rangle_{h} \\
\left\langle\tilde{\psi}_{4}\right\rangle=-\left.\frac{m}{256}\left\langle K_{r r}\left(12 \psi_{1} \psi_{2}-6 \psi_{3}-5 \psi_{1}^{3}\right)\right\rangle\right|_{h} \\
+\frac{7}{8} \int_{m / 2}^{\infty} \frac{\mathrm{d} r^{\prime}}{r^{\prime 2} \psi_{0}^{2}} \int_{\frac{m}{2}}^{r^{\prime}} \frac{\tilde{r}^{2}}{\psi_{0}^{7}}\left\langle K^{2}\left(4 \frac{\psi_{1}^{2}}{\psi_{0}}-\psi_{2}\right)\right\rangle \mathrm{d} \tilde{r} \\
P_{I}^{(4)}=\frac{1}{256 \pi^{2}}\left(\int_{m / 2}^{\infty} \frac{r^{2}\left\langle K^{2}\right\rangle}{\psi_{0}^{6}} \mathrm{~d} r\right)^{2}+\frac{9 m^{4}}{65536 \pi^{2}}\left\langle K_{r r} \psi_{1}\right\rangle_{h}^{2} \\
-\frac{m^{2}}{16 \pi}\left\langle\frac{\psi_{1}^{4}}{4}+6 \psi_{1}^{2} \psi_{2}+6 \psi_{2}^{2}+12 \psi_{1} \psi_{3}\right\rangle_{h}-\frac{3 m^{4}}{16384 \pi^{2}}\left\langle K_{r r}\left(\psi_{2}-\psi_{1}^{2}\right)\right\rangle_{h}\left\langle K_{r r}\right\rangle_{h} \\
+\frac{7 m}{8 \pi} \int_{m / 2}^{\infty} \frac{r^{2}\left(2-\psi_{0}\right)}{\psi_{0}^{8}}\left\langle K^{2}\left(4 \frac{\psi_{1}^{2}}{\psi_{0}}-\psi_{2}\right)\right\rangle \mathrm{d} r \\
+\frac{3 m^{2}\left\langle K_{r r} \psi_{1}\right\rangle_{h}}{2048 \pi^{2}} \int_{m / 2}^{\infty} \frac{r^{2}\left\langle K^{2}\right\rangle}{\psi_{0}^{6}} \mathrm{~d} r+\frac{7 m^{2}\left\langle K_{r r}\right\rangle_{h}}{1024 \pi^{2}} \int_{h}^{\infty} \frac{r^{2}\left\langle K^{2} \psi_{1}\right\rangle}{\psi_{0}^{7}} \mathrm{~d} r .
\end{gathered}
$$


To compute $P_{I}^{(4)}$ we first solve (using Mathematica) the Poisson equation for $\psi_{3}$

$$
\Delta \psi_{3}=\frac{7}{8 \psi_{0}^{8}} K^{2} \psi_{1}
$$

with the boundary condition

$$
\partial_{r} \psi_{3}+\frac{1}{m} \psi_{3}=\frac{3}{64} K_{r r}\left(\psi_{1}^{2}-\psi_{2}\right) \text { at } r=m / 2 .
$$

Correction $\left(P_{I}-p^{2}-\frac{4 \pi}{\left|S_{h}\right|} J^{2}\right)^{(4)}$ for data $103 p$ takes the form of a fourth order homogeneous polynomial $W\left(p / m, \tilde{p} / m^{3}, J / m^{2}\right)$ independent of $a$

$$
\left(P_{I}-p^{2}-\frac{4 \pi}{\left|S_{h}\right|} J^{2}\right)^{(4)}=m^{2} W=0,088 \frac{p^{4}}{m^{2}}+\ldots
$$

If $p \neq 0$ then the term $p^{4}$ dominates in $W$ and we have

$$
W>0,086 \frac{p^{4}}{m^{4}} \text {. }
$$

If $p=0$ then

$$
W \geq 0,004 \frac{\tilde{p}^{4}}{m^{12}}+0,044 \frac{\tilde{p}^{2} J^{2}}{m^{10}}+0,013 \frac{J^{4}}{m^{8}} .
$$

Thus, if any of the parameters $p, \tilde{p}, J$ is nontrivial then the sharp Penrose inequality (12) is satisfied in the fourth order in $K$. If $p=\tilde{p}=J=0$ then data 103 are spherically symmetric, so they should correspond to the Schwarzschild solution. Indeed, this metric admits sections of the form $t=f(r)$ which are conformally equivalent to (103) 22]. Thus, in this case the Penrose inequality is saturated.

\section{Summary and discussion}

Summarizing the last two sections we can formulate our main result in the following way.

Theorem 5.1 Let $S=R^{3} \backslash B\left(0, \frac{m}{2}\right)$ be an initial surface bounded by the sphere $S_{h}$ with radius $m / 2$. Let $g_{i j}$ be flat metric on $S$ and $K_{i j}$ be a traceless axially symmetric solution of the momentum constraint satisfying condition (14). Assume that

- the Lichnerowicz equation (8) admits solution $\psi>0$ satisfying the boundary condition (9) on $S_{h}$ and the asymptotic condition $\psi \longrightarrow 1$

- $\psi$ can be expanded into powers of a parameter $\epsilon$ proportional to a norm of tensor $K_{i j}$.

Then initial data (7) satisfy the Penrose inequality (12) up to the second order in $\epsilon$ in generic case and up to the fourth order in the case of generalized BowenYork data (103). Inequality (12) is saturated only in the case (103) with $p=\tilde{p}=J=0$ which corresponds to the Schwarzschild metric. 
Note that the conformal factor $\psi>0$ is known to exist for $K_{r r}$ of definte sign on $S_{h}$ (see discussion in the begining of Section 2). A more serious problem is lack of a criterion of smallness of $K_{i j}$. Since $K^{2}$ is integrable we can write $K_{i j}=\epsilon \hat{K}_{i j}$, where the integral of $\hat{K}^{2}$ is 1 . If $\psi$ exists and is differentiable with respect to $\epsilon$ we can expand it into the Taylor series in $\epsilon$. Our results correspond to the leading terms $\left(\epsilon^{2}\right.$ or $\left.\epsilon^{4}\right)$ in an expansion of $(12)$. Thus, the Penrose inequality is satisfied for sufficiently small $\epsilon$, but it is difficult to estimate a range of $\epsilon$. In order to have any idea when our approximation agrees with exact results we solved the Lichnerowicz equation numerically in the case 103 with $a=\tilde{p}=0$ using FreeFEM solver [23. We followed an approach in 24], where $r, \theta$ were replaced by coordinates $x=\frac{r}{r+m / 2}$ and $y=\cos \theta$ which cover the finite domain $[1 / 2,1] \times[-1,1]$. Numerical results have been obtained for the specific choice of Schwarzschild mass $(m=2)$ and then generalized to any value of $m$ by a simple rescaling of variables. Their comparison with our perturbative results is shown in Figure 1, where the intensity of the black color corresponds to the difference between expression $\sqrt{116}$ and numerical value of $P_{I}-p^{2}-\frac{J^{2}}{4 \pi\left|S_{h}\right|}$ which is positive in all considered cases. Since this function is independent of sign of $p$ and $J$ it is sufficient to consider only positive values of these parameters. The plot has been obtained by approximating between 273 grid points spaced equidistantly in the domain $[0,1.7] \times[0,3]$ of variables $p / m$ and $J / m^{2}$. It can be seen that, roughly, for $|p|<0,5 m$ and $|J|<2,5 m^{2}$ perturbative formula 116 underestimates a real value of $P_{I}-p^{2}-\frac{J^{2}}{4 \pi\left|S_{h}\right|}$. This situation changes for higher values of $|p|$ and $|J|$.

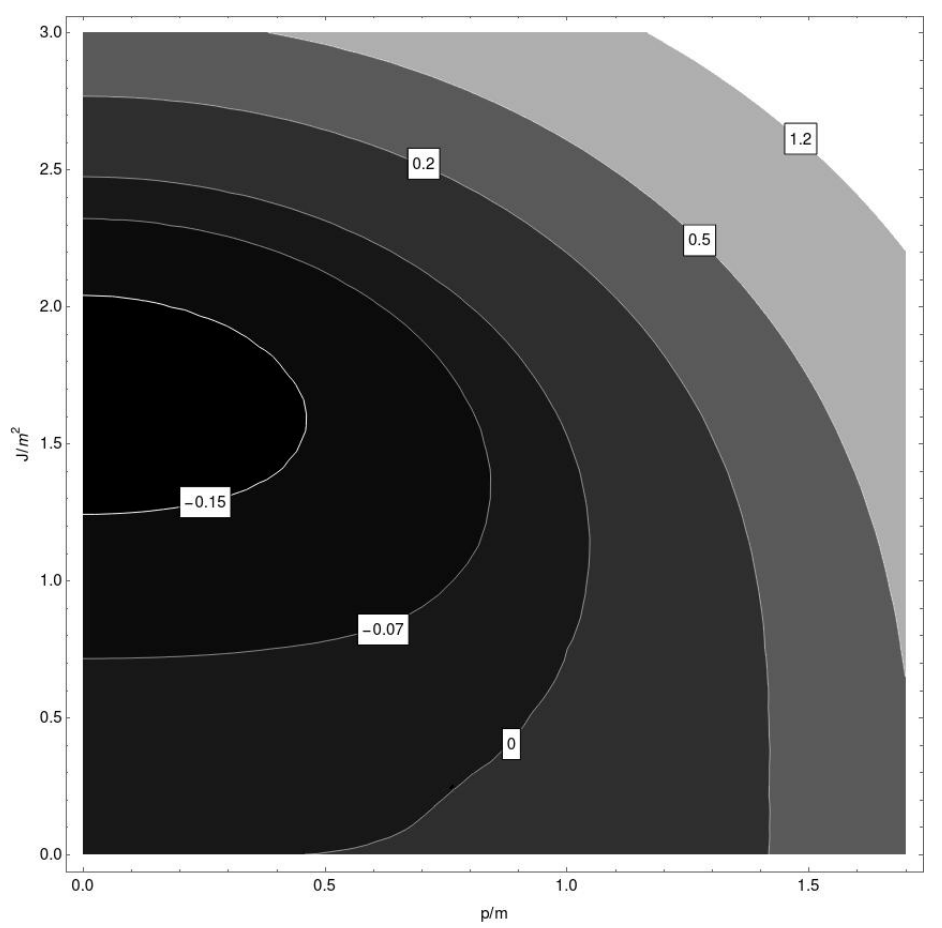

Figure 1: The difference between approximate (up to the fourth order of expansion in $\epsilon)$ and numerical values of $\frac{1}{m^{2}}\left(P_{I}-p^{2}-\frac{J^{2}}{4 \pi\left|S_{h}\right|}\right)$. 
Problems with fixing admitted value of the parameter $\epsilon$ is a disadvantage of our approach when compared to the Geroch method. On the other hand the latter method seems suitable only for the Penrose inequality in the case when the inner boundary is an outermost minimal surface (see [5, 6] for an exception).

Our results concern a class of initial data which depend at most on two free functions of two variables. In order to generalize them within the set of conformally flat data one should admit nonmaximal data $(H \neq 0)$ depending on the azimuthal angle $\varphi$ and horizons which are not spheres. We continue our research in this direction.

\section{References}

[1] Penrose R 1973 Naked singularities Ann. Acad. Sci. 224 125-34

[2] Geroch R 1973 Energy Extraction Ann. Acad. Sci. 224 108-117

[3] Jang P.S. and Wald R.M. 1977 The positive energy conjecture and the cosmic censor hypothesis, J. Math. Phys. 18, 41

[4] Huisken G and Ilmanen T 2001 The inverse mean curvature flow and the Riemannian Penrose inequality J. Diff. Geom. 59 353-437

[5] Malec M., Mars M. and Simon W 2002 On the Penrose Inequality for General Horizons, Phys. Rev. Lett. 88, 121102

[6] Roszkowski K and Malec E. 2005 The Penrose inequality in perturbed Schwarzschild geometries, Acta Phys. Polon. B36, 2931

[7] Mars M. 2009 Present status of the Penrose inequality, Class. Quantum Grav. 26, 193001

[8] Lichnerowicz A. 1944 L'intgration des quations de la gravitation relativiste et le problme des n corps, J. Math. Pures Appl. 23, 39-63

[9] York Jr. J. W. 1971 Gravitational Degrees of Freedom and the Initial-Value Problem, Phys. Rev. Lett. 26, 1656

[10] Dain S. 2004 Trapped surfaces as boundaries for the constraint equations. Class. Quantum Grav., 21(2): 555573

[11] Maxwell D 2005 Solutions of the Einstein constraint equations with apparent horizon boundaries, Commun. Math. Phys. 253 561-583

[12] Maxwell D 2003 Solutions of the Einstein constraint equations with apparent horizon boundaries, arXiv:gr-qc/0307117v1

[13] Dilts J., Holst M., Kozareva T. and Maxwell D. 2017 Numerical Bifurcation Analysis of the Conformal Method, arXiv:1710.03201

[14] Ben-Dov I. 2004 The Penrose inequality and apparent horizons, Phys. Rev. D 70, 124031

[15] Friedman J.L. and Mayer S. 1982 Vacuum handles carrying angular momentum; electrovacuum handles carrying net charge, J. Math. Phys. 23, 109-115 
[16] Bowen J.M and York Jr. J W 1980 Time-asymmetric initial data for black holes and black-hole collisions, Phys. Rev. D21, 2047

[17] Beig R. 2000 Generalized Bowen-York Initial Data. In: Cotsakis S., Gibbons G.W. (eds) Mathematical and Quantum Aspects of Relativity and Cosmology. Lecture Notes in Physics, vol 537. Springer, Berlin, Heidelberg

[18] Conboye R and Murchadha N O 2014 Potentials for transverse trace-free tensors, Class. Quantum Grav. 31, 085019

[19] Conboye R. 2016 Coordinate independent expression for transverse tracefree tensors. Class. Quantum Grav. 33, 015008

[20] Tafel J. 2018 All transverse and TT tensors in flat spaces of any dimension, Gen. Rel. Grav. 50, 31

[21] Tafel J. and Jóźwikowski M. 2017, New solutions of initial conditions in general relativity, Class. Quantum Grav. 31, 115001

[22] Malec E. and Murchadha N. 2003 Constant mean curvature slices in the extended Schwarzschild solution and collapse of the lapse, Part I, Phys.Rev. D68 124019

[23] Hecht F. 2012 New development in FreeFem++. Journal of numerical mathematics, 20(3-4), 251-266

[24] Karkowski J. and Malec E. 2005 The General Penrose Inequality: Lessons from Numerical Evidence, Acta Phys.Polon. B36 59-74 